\title{
Numerical Simulation of Fuzzy Wave-Like Equations with Spatial Variable Coefficients by Lattice Boltzmann Method
}

\author{
Zongning ZHANG ${ }^{\mathrm{a}, \mathrm{b}}$ and Jianqiang $\mathrm{DONG}^{\mathrm{a}, 1}$ \\ a School of Mathematics and Information Science, North Minzu University, Ningxia, \\ Yinchuan, 750021, China \\ ${ }^{\mathrm{b}}$ Zhengzhou University of Science and Technology, Zhengzhou, Henan 450000, China
}

\begin{abstract}
In this paper, lattice Boltzmann model is used to simulate a class of nonlinear fuzzy wave-like equations with spatial variable coefficients. we recovers the fuzzy wave-like equations by designing the equilibrium state distribution function and using the Chapman-Enskog multi-scale technology. Theoretically, it proves that the computing model is compatible with fuzzy wave-like equations. And based on this, the author further verifies the feasibility and effectiveness of the constructed lattice Boltzmann model by using numerical examples.
\end{abstract}

Keywords. Lattice Boltzmann method, fuzzy wave-like equation, numerical simulations

\section{Introduction}

The lattice Boltzmann method (LBM)[1-2], as an effective numerical approach, has achieved success in simulating some complex fluid flows. Recently, LBM has been extended successfully to simulate some nonlinear evolution equations(NLEEs), such as , KdV-Burgers equation with variable coefficients[1], Fokker-Planck equation with [3], a class of convection-diffusion equations with variable coefficients[4], generalized Gardner equation with time dependent variable coefficients[5-6] and so on.

Unlike traditional macroscopic numerical method that directly discretize the control equations in time and space, LBM is a mesoscopic numerical calculation method. The LB equation is based on a basic discrete velocity dynamics equation, which includes particle collision and propagation, these two processes can clearly restore various macroscopic physical phenomena. We note that the model in references [7] can be used to solve the Navier-Stokes and nonlinear convection-diffusion equations, In references [8] give two complementary lattice Boltzmann models that asymptotically reproduce solutions of nonlinear systems are derived by the CE method.

In this paper, $\mathrm{CE}$ analysis method is used to obtain the macroscopic fuzzy wavelike equations. We assume the Knudsen number $\varepsilon$ as the time step $\Delta t$. Based on the previous works, we develop a LB model for a fuzzy wave-like equations with variable

\footnotetext{
${ }^{1}$ Corresponding Author, Jianqiang DONG, School of Mathematics and Information Science, North Minzu University, Ningxia, Yinchuan, 750021, China; Email: djq860707@163.com.
} 
coefficients. Here, we consider the $1+1$ dimensions wave-like equation which can be written as the following form.

$$
U_{t t}+p(x) \Delta U=F(x, t, k)
$$

Eq.(1) can describe earthquake stresses and non-homogeneous elastic waves in soils. In this equation, $\mathrm{U}$ is the macro variables, the coefficients $p(x)$ is analytic function of space $x, F(t, x, k)$ is source term and $\Delta$ is the laplacian.

This paper is organized as follows: In Section(2), It proves that the microscopic lattice Boltzmann equation is compatible with the macroscopic equation. In Section(3), We use LBM to do some numerical experiments on variable coefficient fuzzy wave equations. Finally, we plan to summarize the whole article and give the steps to use the LBM method to solve partial differential equations in Section(4).

\section{The Lbgk Model for the Fuzzy Wave-Like Equations}

In this work, the single-relaxation-time BGK model is considered. The evolution equation of the SRT-LB model reads,

$$
f_{\alpha}\left(x+e_{\alpha} \Delta t, t+\Delta t\right)-f_{\alpha}(x, t)=-\frac{1}{\tau}\left[f_{\alpha}(x, t)-f_{\alpha}^{e q}(x, t)\right]+\Delta t \frac{F(x, t, k)}{q}, \quad \alpha=0,1, \cdots q-1
$$

where $e_{\alpha}$ is the lattice speed and $\tau$ is the dimensionless relaxation time, $f_{\alpha}(x, t)$ is the distribution function of particles, $f_{\alpha}^{e q}(x, t)$ is the local equilibrium distribution function of particles, $F(x, t, k)$ stands for source term with variable coefficient, $q$ is the number of grid speeds, Respectively.

We define the macro parameter $\mathrm{v}$ as the following form,

$$
v(x, t)=\frac{\partial u(x, t)}{\partial u}=\sum_{\alpha=0}^{q-1} f_{\alpha}(x, t)=\sum_{\alpha=0}^{q-1} f_{\alpha}^{e q}(x, t)
$$

The Chapman-Enskog expansion is applied to $f_{\alpha}(x, t)$, we have

$$
f_{\alpha}=f_{\alpha}^{(0)}+\varepsilon f_{\alpha}^{(1)}+\varepsilon^{2} f_{\alpha}^{(2)}+\cdots
$$

at the same time, we expand the time term, space term, and source term by CE, we can get, 


$$
\begin{aligned}
& \partial_{t}=\varepsilon \partial_{t_{1}}+\varepsilon^{2} \partial_{t_{2}}+O\left(\varepsilon^{3}\right), \\
& \nabla=\varepsilon \nabla_{1}, \\
& F(x, t, k)=\varepsilon F_{1}(x, t, k) .
\end{aligned}
$$

We directly use Taylor expansion for the left term of Eq.(2),

$$
\Delta t D_{\alpha} f_{\alpha}+\frac{\Delta t^{2}}{2 !} D_{\alpha}^{2} f_{\alpha}+\frac{\Delta t^{3}}{3 !} D_{\alpha}^{3} f_{\alpha}+\cdots=-\frac{1}{\tau}\left(f_{\alpha}-f_{\alpha}^{e q}\right)+\Delta t \frac{F(x, t, k)}{q}, \quad \mathrm{q}=0,1, \cdots \mathrm{q}-1
$$

where $D_{\alpha}=\partial_{t}+c_{\alpha} \cdot \nabla, D_{1, \alpha}=\partial_{t_{1}}+c_{i} \cdot \nabla_{1}$. Substituting Eqs.(4), (5) into Eq.(6), we can obtain,

$$
\begin{gathered}
O\left(\varepsilon^{0}\right):-\frac{1}{\tau}\left(f_{\alpha}^{(0)}-f_{\alpha}^{e q}\right)=0 \\
O\left(\varepsilon^{1}\right): D_{1, \alpha} f_{\alpha}^{(0)}=-\frac{1}{\tau \Delta t} f_{\alpha}^{(1)}+\frac{F_{1}(x, t, k)}{q}, \\
O\left(\varepsilon^{2}\right): \partial_{t_{2}} f_{\alpha}^{(0)}+D_{1, \alpha} f_{\alpha}^{(1)}+\frac{\Delta t}{2} D_{1 \alpha}^{2} f_{\alpha}^{(0)}=-\frac{1}{\tau \Delta t} f_{\alpha}^{(2)},
\end{gathered}
$$

Based on the fact that $\tau>0.5$ [4], Eq.(8) reads

$$
f_{\alpha}^{(0)}=f_{\alpha}^{e q}, \quad \mathrm{q}=0,1, \cdots \mathrm{q}-1 .
$$

With the help of Eq.(8),we can rewrite Eq.(9) as

$$
\partial_{t_{2}} f_{\alpha}^{(0)}+\Delta t\left(\frac{1}{2}-\tau\right) D_{1, \alpha}^{2} f_{\alpha}^{(0)}=-\frac{1}{\tau \Delta t} f_{\alpha}^{(2)}
$$

From Eqs(3), (4), and (10), we have,

$$
\sum_{\alpha=0}^{\mathrm{q}-1} f_{\alpha}^{(k)}=0 \quad(k \geq 1)
$$

in order to restore the macro Eq. (1), we make the following constraints on the equilibrium function, 


$$
\left\{\begin{array}{l}
\sum_{\alpha=0}^{\mathrm{q}-1} c_{\alpha} f_{\alpha}^{(0)}=0 \\
\sum_{\alpha=0}^{\mathrm{q}-1} c_{\alpha}^{2} f_{\alpha}^{(0)}=\frac{P(x) v}{\Delta t(\tau-0.5)}
\end{array}\right.
$$

summing Eq.(8) over $\alpha$ and using Eqs.(3), (12)and (13),we can obtain,

$$
\frac{\partial v}{\partial t_{1}}+\frac{\partial}{\partial x_{1}}\left(\sum_{\alpha=0}^{\mathrm{q}-1} c_{\alpha} f_{\alpha}^{(0)}\right)=\sum_{\alpha=0}^{\mathrm{q}-1} \frac{F(x, t, k)}{q}
$$

similarly, summing Eq.(11) over $\alpha$, and using Eqs.(3), (12) and (13),

$$
\frac{\partial v}{\partial t_{2}}+\Delta t\left(\frac{1}{2}-\tau\right) \sum_{\alpha=0}^{\mathrm{q}-1} D_{1, \alpha}^{2} f_{\alpha}^{(0)}+O\left(\varepsilon^{3}\right)=0
$$

according to Eqs.(3), (11) and (12), we have

$$
\begin{aligned}
\sum_{\alpha=0}^{\mathrm{q}-1} D_{1, \alpha}^{2} f_{\alpha}^{(0)} & =\frac{\partial^{2}}{\partial t_{1}^{2}}\left(\sum_{\alpha=0}^{\mathrm{q}-1} f_{\alpha}^{(0)}\right)+2 \frac{\partial^{2}}{\partial t_{1} \partial x_{1}}\left(\sum_{\alpha=0}^{\mathrm{q}-1} c_{i} f_{\alpha}^{(0)}\right)+\frac{\partial^{2}}{\partial x_{1}^{2}}\left(\sum_{\alpha=0}^{\mathrm{q}-1} c_{i}^{2} f_{\alpha}^{(0)}\right) \\
& =\frac{1}{\Delta t(\tau-0.5)} \frac{\partial^{2}}{\partial x_{1}^{2}}[P(x) v],
\end{aligned}
$$

substituting to Eq.(16) into Eq.(15) yields,

$$
\frac{\partial v}{\partial t_{2}}+\frac{\partial^{2}}{\partial x_{1}^{2}}[P(x) v]+O\left(\varepsilon^{3}\right)=0
$$

by taking $E q \cdot(14) \times \varepsilon+E q \cdot(17) \times \varepsilon^{2}$,

$$
\frac{\partial v}{\partial t}+p(x) v+F(x, t, k)=O\left(\varepsilon^{3}\right)
$$

Eq.(18) is the same as Eq.(1) in mathematical form. Based on Eq.(3), (14), The 5bit equilibrium function we constructed is, 


$$
\left\{\begin{array}{l}
f_{0}^{e q}=v-\frac{5 P(x) u}{4 c^{2} \Delta t(\tau-0.5)}, \\
f_{1}^{e q}=f_{2}^{e q}=\frac{2 P(x) u}{3 c^{2} \Delta t(\tau-0.5)}, \\
f_{3}^{e q}=f_{4}^{e q}=-\frac{P(x) u}{24 c^{2} \Delta t(\tau-0.5)} .
\end{array}\right.
$$

For the item $v=\mathrm{u}_{t}$, we have

$$
u(x, t)=\Delta t \sum_{\alpha=0}^{q-1} f_{\alpha}^{e q}(x, t)+u(x, t-\Delta t)
$$

Remark: In Eq.(18) , $\quad p(x)$ and $P(x)$ satisfy: $\bar{p}(x)=\int p(x) d x, P(x)=\int \bar{p}(x) d x$.

\section{Numerical Simulation}

In this section we do some numerical experiments, and use the following error indicators to verify the effectiveness of our model. The non-equilibrium extrapolation scheme proposed by Guo et al [9] is used for the boundary treatments.

1. $L_{\infty}$ error

$$
L_{\infty}=\max _{i=1,2, \cdots N}\left|u\left(x_{i}, t\right)-u^{*}\left(x_{i}, t\right)\right|,
$$

2. $L_{2}$ error

$$
L_{2}=\frac{1}{N} \sqrt{\sum_{i=1}^{N}\left[u\left(x_{i}, t\right)-u^{*}\left(x_{i}, t\right)\right]^{2}},
$$

3. The root mean square error 


$$
\mathrm{RMS}=\sqrt{\sum_{i=0}^{N} \frac{\left[u\left(x_{i}, t\right)-u^{*}\left(x_{i}, t\right)\right]^{2}}{N+1}},
$$

4. The global relative error

$$
\mathrm{GRE}=\frac{\sum_{i=1}^{N}\left|u\left(x_{i}, t\right)-u^{*}\left(x_{i}, t\right)\right|}{\sum_{i=1}^{N}\left|u^{*}\left(x_{i}, t\right)\right|}
$$

Example For $p(x)=x$, Eq.(1) becomes

$$
U_{t t}(t, x)+x U_{x x}(t, x)=-k x^{2}
$$

with the initial conditions $U(0, x)=c x^{2}, \quad U_{t}(0, x)=1$, where $x \in[-1,1]$, $t \in[0,1], k, c$ are arbitrary non-negative constant . The exact solution is[10]

$$
U(x, t)=\frac{1}{12} \mathrm{kxt}^{4}-\frac{1}{2} \mathrm{kx}^{2} \mathrm{t}^{2}+c x^{2}-c x t^{2}+t
$$

We take $k=c=1$, table 1 shows the numerical results of $L_{\infty}, L_{2}$, RMS, GRE , CPU at different moments. Figure 1(a) shows the comparisons of the numerical solutions and exact results where $\Delta x=0.1, \Delta t=0.001, \tau=1$, figure 1(b) shows the image of the LBM results in three dimensional space.

Table 1. Errors of the model for Example at different time

\begin{tabular}{cccccc}
\hline$t$ & $L_{\infty}$ & $L_{2}$ & $R M S$ & GRE & $C P U$ times(s) \\
\hline$t=0.4$ & 0.2233 & 0.3286 & 0.0327 & 0.0029 & 0.1834 \\
$t=0.6$ & 0.2524 & 0.3188 & 0.0317 & 0.0027 & 0.1823 \\
$t=0.8$ & 0.2919 & 0.3206 & 0.0319 & 0.0025 & 0.1747 \\
$t=1.0$ & 0.3418 & 0.3490 & 0.0347 & 0.0018 & 0.1819 \\
\hline
\end{tabular}




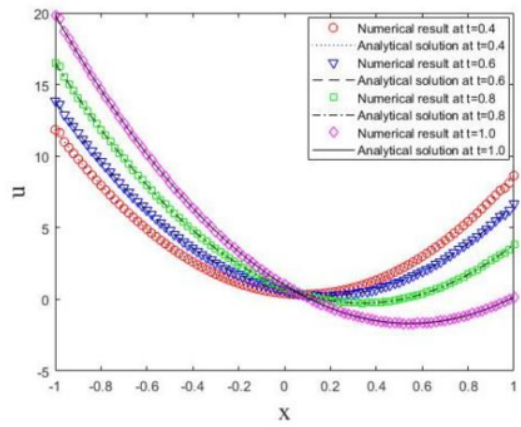

(a)

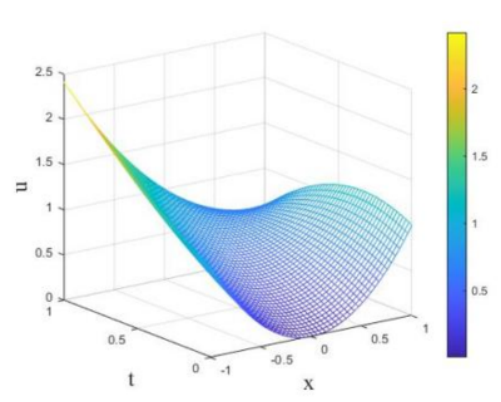

(b)

Figure 1. (a)Numerical result and analytical solution at $\mathrm{t}=0.4,0.6,0.8,1.0$

(b)The flowchart of our numerical simulation procedures

\section{Conclusion}

This paper studies the lattice Boltzmann model of a class of generalized fuzzy wavelike equations with spatial variable coefficient. Firstly, the Eq.(1) is established by selecting the balanced distribution function. The D1Q5 lattice Boltzmann model is used to carry out numerical simulation experiments on different forms of fuzzy wave-like equations. Secondly, it is proved that the lattice Boltzmann model is compatible with the macroscopic equation. Finally, The model is extended to higher-dimensional variable coefficient fuzzy wave-like equations, therefor, the present model may be more useful in the study of such problems.

\section{Acknowledgement}

This article was supported by the Natural Science Foundation of Ningxia(2021AAC03206) and the Postgraduate Innovation Project of North Minzu University (YCX21156).

\section{References}

[1] Zongning Zhang, Chunguang Li, Jianqiang Dong. General Propagation Lattice Boltzmann Model for a Variable-Coefficient Compound KdV-Burgers Equation[J].Acta Mathematica Scientia. 2021,41(A), 5: 1283-1295.

[2] Succi S. The lattice Boltzmann equation for fluid dynamics and beyond[J]. Phys Today. 2002, 55(12): 58-60.

[3] Yan GW. A lattice Boltzmann equation for waves[J]. J Comput Phys.2000, 161(1):61-69.

[4] Zhang CY, Tan HL, Liu MR, et al. A lattice Boltzmann model and simulation of KdV-Burgers equation[J]. Commun Theor Phys. 2004, 42(2): 281-284.

[5] Li QH, Chai ZH, Shi BC. Lattice Boltzmann model for a class of convection-diffusion equations with variable coefficients[J]. Comput Math Appl. 2015;70(4): 548-561. 
[6] Hu WQ, Gao YT, Lan ZZ. Lattice Boltzmann model for a generalized Gardner equation with timedependent variable coefficients[J]. Appl Math Mod. 2017, 46:126-140.

[7] Chai ZZ, Shi BC. Multiple-relaxation-time lattice Boltzmann method for the Navier-Stokes and nonlinear convection-diffusion equations:Modeling,analysis,and elements[J]. Phys Rev E. 2020, 102:02336.

[8] Otomo H, Boghosian BM, Dubois F. Two complementary lattice-Boltzmann-based analyses for nonlinear systems[J]. Phys A.2017, 486:1000-1011.

[9] Guo ZL, Zheng CG, Shi BC. Non-equilibrium extrapolation method for velocity and pressure boundary conditionsin the lattice Boltzmann method[J]. Chin Phys. 2002, 11:366-374.

[10] Allahviranloo T, Abbasbandy S, Rouhparvar H. The exact solutions of fuzzy wave-like equations with variable coefficients by a variational iteration method[J]. Appl Soft Comput.2011, 11:2186-2192. 